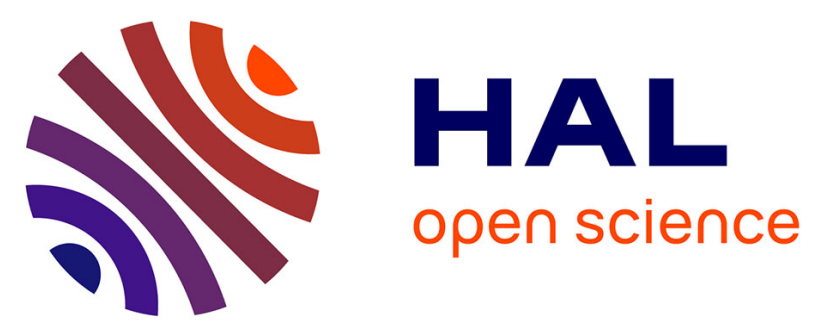

\title{
Parametric X-ray radiation for calibration of X-ray space telescopes and generation of several X-ray beams
}

\author{
A.V. Shchagin, N.A. Khiznyak, R.B. Fiorito, D.W. Rule, X. Artru
}

\section{To cite this version:}

A.V. Shchagin, N.A. Khiznyak, R.B. Fiorito, D.W. Rule, X. Artru. Parametric X-ray radiation for calibration of X-ray space telescopes and generation of several X-ray beams. Nuclear Instruments and Methods in Physics Research Section B: Beam Interactions with Materials and Atoms, 2001, 173, pp.154-159. 10.1016/S0168-583X(00)00059-8 . in2p3-00024744

\section{HAL Id: in2p3-00024744 \\ https://hal.in2p3.fr/in2p3-00024744}

Submitted on 23 Jun 2021

HAL is a multi-disciplinary open access archive for the deposit and dissemination of scientific research documents, whether they are published or not. The documents may come from teaching and research institutions in France or abroad, or from public or private research centers.
L'archive ouverte pluridisciplinaire HAL, est destinée au dépôt et à la diffusion de documents scientifiques de niveau recherche, publiés ou non, émanant des établissements d'enseignement et de recherche français ou étrangers, des laboratoires publics ou privés. 


\title{
Parametric X-ray radiation for calibration of X-ray space telescopes and generation of several X-ray beams
}

\author{
A.V. Shchagin ${ }^{a, *}$, N.A. Khizhnyak ${ }^{a}$, R.B. Fiorito ${ }^{b}$, D.W. Rule ${ }^{c}$, X. Artru ${ }^{\text {d }}$ \\ ${ }^{a}$ Kharkov Institute of Physics and Technology, Alcademicheskaya Str. 1, Kharkov 310108, Ukraine \\ ${ }^{\mathrm{b}}$ Department of Physics, Catholic University of America, 620 Michigan Ave. NE, Washington, DC 20064, USA \\ ${ }^{c}$ Carderock Division, Naval Surface Warfare Center, Code 682, 9500 MacArthur Boulevard, West Bethesda, MD 20817-5700, USA \\ ${ }^{\mathrm{d}}$ Institut de Physique Nucleaire de Lyon, IN2P3-CNRS and Universite Claude-Bernard, 43 Boulevard du 11 Novembre 1918, \\ F-69622 Villeurbanne cedex, France
}

\begin{abstract}
The setup based on a moderate energy linear accelerator (linac) is proposed to provide users with a tunable, quasimonochromatic, linearly polarized X-ray beam. The effect of parametric X-ray radiation (PXR) from relativistic electrons in a crystal is used in an X-ray source. The application of the setup for calibration of X-ray space telescopes and other equipment is considered. The setup allows calibration of angular, spectral, polarization parameters of telescopes with smooth tuning of X-ray energy from several $\mathrm{keV}$ to hundreds of keV. Estimates of X-ray flux intensities and spectral line widths on the telescope aperture at a distance of several hundred meters are presented. Besides, the PXR-based facility for simultaneous generation of several X-ray beams is proposed.
\end{abstract}

Keywords: Parametric X-ray radiation (PXR); X-ray beam; Calibration; X-ray space telescope

\section{Introduction}

Following first theoretical predictions [1,2], the parametric X-ray radiation (PXR) was observed experimentally in 1985 [3]. Then, the basic PXR

\footnotetext{
${ }^{*}$ Corresponding author. Tel.: +380-572-35-68-84; fax: +380572-35-35-64.

E-mail address: shchagin@kipt.kharkov.ua (A.V. Shcha-
}

properties were studied by several groups (see, e.g., [4-7]). For more detailed descriptions of PXR phenomenon, properties and investigations performed until 1996, we refer the reader to the review [8]. References for a few more recent papers are listed in [9]. The PXR polarization has been considered in [10].

The PXR is a quasi-monochromatic linearly polarized X-ray radiation generated by a relativistic charged particle moving through a crystal. The angular distribution of PXR yield shows 
sharp maxima (so-called PXR reflection) in the vicinity of the Bragg direction. The angular distance between the Bragg direction and the maxima (angular size of PXR reflection) is usually close to $\gamma_{\text {eff }}^{-1}$, where $\gamma_{\text {eff }}$ is the effective relativistic factor of incident particle in the crystal [10]. The PXR spectral peak can have a moderate width at a low spectral background. Experimentally, the PXR spectral peak has been observed at energies from several $\mathrm{keV}$ up to about $400 \mathrm{keV}$. The PXR properties allow one to consider this radiation as an alternative to a synchrotron source of a hard $\mathrm{X}$-ray beam, especially at energies higher than a few tens of keV [11].

The PXR spectral peak energy can be smoothly tuned by simple rotation of a crystal radiator placed in the particle beam. However, the direction of PXR reflection emission changes with this rotation, too. Therefore, the direction of maxima in the angular PXR yield distribution changes relative to the particle beam as the X-ray energy is tuned. In this paper, we propose a scheme of experimental setup for wide-range tuning of spectral peak energy in a fixed PXR reflection. As an example, we will consider the PXR applicability for calibration of X-ray space telescopes and/or other $\mathrm{X}$-ray equipment in the energy range from several $\mathrm{keV}$ up to hundreds of $\mathrm{keV}$, first briefly indicated in $[12,13]$.

Besides, the setup can provide simultaneous generation of several tunable X-ray beams directed to several fixed beamlines. We propose the scheme of a multi-X-ray beam facility that can be used for various applications.

\section{Experimental setup}

In principle, there are several possibilities to provide the maximum PXR yield with energy tunable in a wide range for a fixed X-ray beamline at an immobile accelerator. One of them can be realized through a change in the particle beam direction by bending magnet(s). Another possibility can be realized by reflecting the PXR to the $\mathrm{X}$-ray beamline using an X-ray mirror(s). Here, we describe the most obvious scheme, where the whole accelerator is placed on a rotating platform to provide a tunable angle of particle beam incidence relative to the fixed $\mathrm{X}$-ray beamline axis.

The scheme of the setup is shown in Fig. 1. The setup includes an X-ray beamline BL in a vacuum tube with collimators, a crystal radiator $\mathrm{C}$, an electron linear accelerator (linac) $\mathrm{L}$ placed on a rotating platform. The X-ray consumer $\mathrm{T}$ (for example, X-ray telescope) is at the end of the beamline. The setup plane is determined by the axes of the X-ray beamline and the linac. The cleaning magnet $\mathrm{M}$ declines the electrons scattered from the crystal radiator toward the X-ray beamline. The beamline can be a few hundred meters long to provide a small X-ray beam divergence at the consumer aperture of appreciable size. $\theta$ is the angle between the electron beam axis (or, in other words, the linac axis) and the X-ray beamline axis. This angle can be varied due to rotation of the platform with the linac arranged on it. $\phi$ is the angle between the electron beam axis and the crystallographic planes of the crystal radiator. The crystallographic planes are shown by hatches and are denoted by the reciprocal lattice vector $\vec{g}$.



Fig. 1. The setup for calibration of space X-ray telescopes and for other applications using the PXR-based X-ray beam. T space X-ray telescope at calibration (or other consumer). BL vacuum tube with collimators, passing the $\mathrm{X}$-ray beam from the crystal radiator to the telescope (X-ray beamline). To provide the required angular resolution, the tube can be several hundred meters long. $\mathrm{M}$ - cleaning magnet declining the electrons scattered from the crystal radiator toward the X-ray beamline. $\mathrm{C}-$ crystal radiator installed in the goniometer. The reciprocal lattice vector $\vec{g}$ denotes crystallographic planes shown by parallel lines on the crystal radiator. The centers of both the goniometer and the crystal radiator are at the point of intersection of the axes of the X-ray beamline and the electron beam from linac L. L - moderate energy linac installed on the rotating platform at a tunable angle $\theta$ relative to the $\mathrm{X}$-ray beamline. The tunable angle $\phi$ between crystallographic planes and the electron beam axis is provided by the goniomenter, where the crystal radiator is placed. 
The angle $\phi$ and the crystal-radiator alignment can be changed using the vacuum goniometer, where the crystal radiator is mounted. The goniometer, rotating platform, vacuum system and other technical systems are not shown in Fig. 1.

The electron beam from the linac passes through a crystal-radiator plate. The Laue or Bragg geometry can be used for generation of PXR reflection from the crystallographic planes of the crystal radiator. Fig. 1 shows the Laue geometry case. A certain angular part of PXR reflection reaches the consumer aperture. To direct the X-ray beam of certain energy and maximum intensity along the X-ray beamline axis, both the angles $\theta, \phi$ should be found from Eqs. (9.5) and (9.8) of [8]. For the polarization direction parallel to the setup plane (parallel polarization), we usually have

$\theta \approx 2 \phi \pm \gamma_{\mathrm{eff}}^{-1}$

The present calculations were performed for the sign "_" in this formula. The polarization directions on a small (relative of PXR reflection size) consumer aperture are nearly parallel to the setup plane, provided that the vector $\vec{g}$ is in the same plane [10].

\section{X-ray telescope calibration}

We estimated the X-ray beam properties using the kinematic PXR theory [2], as this theory was confirmed by experiments (e.g., see references in [8]). Eqs. (9.5), (9.7) and (9.8) from [8] were used in our calculations. The crystallographic plane (1 111$)$ of a popular silicon crystal was used for generation of PXR reflection by the relativistic electron beam. This plane is convenient to ensure a low spectral background for telescope calibration due to both the suppression of PXR reflection having a twofold energy and a weak influence of the row effect $[12,13]$. A moderate crystal-radiator thickness of $60 \mu \mathrm{m}$ was chosen to provide moderate electron scattering. In calculations, we used an electron beam current of $100 \mu \mathrm{A}$ and an X-ray beamline length of $1700 \mathrm{ft}(518.16 \mathrm{~m})$, this corresponding to the length of the X-ray calibration facility for calibration of X-ray telescopes at MSFC. ${ }^{1}$

The angular size of the PXR reflection can be estimated as $\gamma_{\text {eff }}^{-1}$. For a $\mathrm{Si}$ crystal, this is about $5 \mathrm{mrad}$ at an electron beam energy $E_{\mathrm{e}}$ of about 100 $\mathrm{MeV}$ and a spectral peak energy $E_{\gamma}$ satisfying the inequality $E_{\gamma}^{2} \gg(6 \mathrm{keV})^{2}$. The angular part of the PXR reflection passing through vacuum tube collimators can provide an X-ray beam diameter up to about $2 \mathrm{~m}$ at the end of the beamline. This diameter can be increased (reduced) by decreasing (increasing) the electron beam energy. The linear polarization directions of the X-ray beam are close to parallel in the vicinity of the setup plane at any value of $\theta, \phi$, provided that the vector $\vec{g}$ is in the same plane. This average direction of polarization can be changed by choosing the vector $\vec{g}$ alignment outside the setup plane.

Some results calculated at an electron beam energy of $120 \mathrm{MeV}$ are given in Table 1. The X-ray flux intensity as a function of spectral peak energy $E_{\gamma}$ is shown in Fig. 2 for electron beam energies of 60, 120 and $180 \mathrm{MeV}$. A strong dependence of flux intensity on the electron beam energy is seen in Fig. 2. It can also be seen that the X-ray flux intensity decreases and the X-ray spectral line width increases as the X-ray energy increases. Generally, the data in Table 1 and Fig. 2 demonstrate the applicability of the setup for the calibration of Xray space telescopes at energies up to hundreds of $\mathrm{keV}$. These data can be used for preliminary estimations in the design of the setup.

The X-ray beam diameter and divergence for a particular telescope aperture size can be provided by a proper choice of electron beam energy and Xray beamline length values. The X-ray flux intensity can be controlled by varying the electron beam current and the crystal-radiator thickness. The calibration of telescope effective registration area can be performed by smoothly tuning the X-ray energy through a smooth adjustment of angles $\theta$, $\phi$. The telescope angular characteristics can be

\footnotetext{
${ }^{1}$ An information on X-ray Calibration Facility for calibration of X-ray telescopes at Marshall Space Flight Center can be found in the site: http://sail.msfc.nasa.gov/xcfdoc/xAhome.html.
} 
Table 1

Properties of polarized X-ray beam at the telescope (or other consumer) aperture for the setup shown in Fig. 1 at a distance of $1700 \mathrm{ft}$ $(518.16 \mathrm{~m})$ for an incident electron beam energy of $120 \mathrm{MeV}$ and a current of $100 \mu \mathrm{A}^{\mathrm{a}}$

\begin{tabular}{lllllll}
\hline $\begin{array}{l}\theta \\
(\mathrm{rad})\end{array}$ & $\begin{array}{l}\phi \\
(\mathrm{rad})\end{array}$ & $\begin{array}{l}E_{\gamma} \\
(\mathrm{keV})\end{array}$ & $\begin{array}{l}\Delta E_{\gamma} / E_{\gamma} \\
(\%)\end{array}$ & $\begin{array}{l}\Delta E_{\gamma} \\
(\mathrm{keV})\end{array}$ & $\begin{array}{l}Y \\
(\text { quanta/electron } \times \text { steradian })\end{array}$ & $\begin{array}{l}F \\
\left(\text { quanta } / \mathrm{s} \times \mathrm{cm}^{2}\right)\end{array}$ \\
\hline 0.300 & 0.1524 & 13.436 & 0.13 & 0.017 & $0.7827 \times 10^{-2}$ & 1820 \\
0.200 & 0.1022 & 20.228 & 0.19 & 0.039 & $0.6581 \times 10^{-2}$ & 1530 \\
0.150 & 0.0772 & 27.135 & 0.26 & 0.070 & $0.5282 \times 10^{-2}$ & 1228 \\
0.100 & 0.0522 & 41.221 & 0.39 & 0.159 & $0.3587 \times 10^{-2}$ & 380 \\
0.050 & 0.0272 & 85.432 & 0.77 & 0.66 & $0.1633 \times 10^{-2}$ & 211 \\
0.032 & 0.0182 & 138.134 & 1.21 & 1.67 & $0.9081 \times 10^{-3}$ & 148 \\
0.025 & 0.0148 & 182.015 & 1.55 & 2.82 & $0.6353 \times 10^{-3}$ & 104 \\
0.020 & 0.0124 & 234.628 & 1.93 & 4.53 & $0.4470 \times 10^{-3}$ & 71 \\
0.016 & 0.0106 & 306.014 & 2.42 & 7.40 & $0.3035 \times 10^{-3}$ & 55 \\
0.014 & 0.0096 & 354.817 & 2.76 & 9.78 & $0.2368 \times 10^{-3}$ & 40 \\
0.012 & 0.0086 & 419.847 & 3.22 & 13.54 & $0.1736 \times 10^{-3}$ & \\
\hline
\end{tabular}

${ }^{\text {a Angles }} \theta, \phi$ are shown in Fig. 1, $E_{\gamma}$ is the X-ray spectral peak energy, $\Delta E_{\gamma}$ is the spectral peak width for a $10 \mathrm{~cm}$ consumer aperture in the setup plane, $Y$ is the PXR yield from the crystal radiatior, $F$ is the $\mathrm{X}$-ray flux intensity at the telescope aperture. Calculations were performed for a Si crystal radiator, $60 \mu \mathrm{m}$ thick, kept up at room temperature, the Laue geometry, $\vec{g}=\langle 111\rangle$.

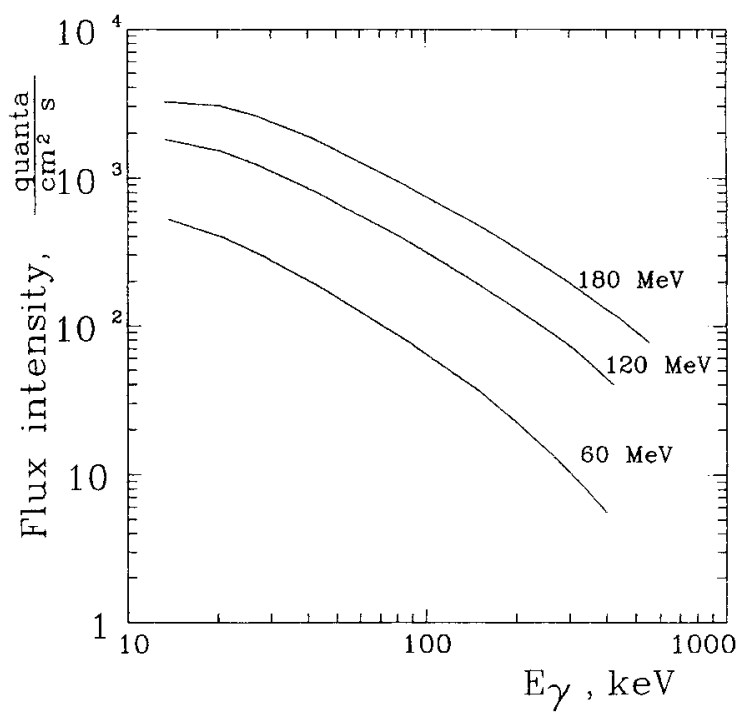

Fig. 2. X-ray flux intensity at the telescope aperture in units of quanta number per $\mathrm{cm}^{2}$ per second as a function of quanta energy for incident electron energies of 60,120 and $180 \mathrm{MeV}$. Calculations were made for a distance of $1700 \mathrm{ft}(518.16 \mathrm{~m})$ between the telescope aperture and the crystal radiator, electron beam current of $100 \mu \mathrm{A}$, Si crystal-radiator thickness of $60 \mu \mathrm{m}$, the Laue geometry, $\vec{g}=\langle 111\rangle$, the crystal radiator is kept at room temperature. The angular size of PXR reflection is of about $8.5,4.3$ or $2.8 \mathrm{mrad}$ at an electron beam energy of 60,120 or $180 \mathrm{MeV}$, respectively. calibrated as functions of telescope alignments. The calibration of the telescope polarization sensitivity at any available energy can be performed in two ways: (i) by rotating the telescope around the $\mathrm{X}$-ray beamline axis at a fixed crystal radiator, or (ii) by changing the X-ray beam polarization direction through a proper alignment of the reciprocal lattice vector $\vec{g}$ outside the setup plane at a fixed telescope. The monitoring of X-ray beam parameters can be performed by the detectors placed in the vicinity of the telescope and/or in the vacuum tube, and also by a control of electron beam parameters. The electron beam power (10 $\mathrm{kW}$ at an energy of $100 \mathrm{MeV}$ and a current of 100 $\mu \mathrm{A}$ ) is comparable to a power of two $18 \mathrm{~kW}$ rotating anode sources used in the X-ray Calibration Facility at MSFC. ${ }^{1}$

\section{Multi-X-ray beam facility}

As mentioned above, we have considered the PXR coming from one set of crystallographic planes only. However, crystals usually have numerous sets of crystallographic planes. A variety of PXR reflections can be generated in the vicinity of their Bragg directions. In the general case, the $\mathrm{X}$-ray energies, yields and Bragg directions vary 
with crystal rotation. The rotation of Bragg directions together with the associated PXR reflections hampers the effective use of several PXR reflections as tunable $\mathrm{X}$-ray beams at immovable linac.

The setup shown in Fig. 1 has a remarkable property, namely, at rotations provided at $\Delta \theta=2 \Delta \phi$ some of PXR reflections stay in the vicinity of the immovable Bragg directions, despite the change in energy and intensity of the reflections. This is true for crystallographic planes perpendicular to the setup plane or, in other words, for reciprocal lattice vectors in the setup plane. This property allows the development of a tunable multi-X-ray beam facility with fixed beamlines for several users.

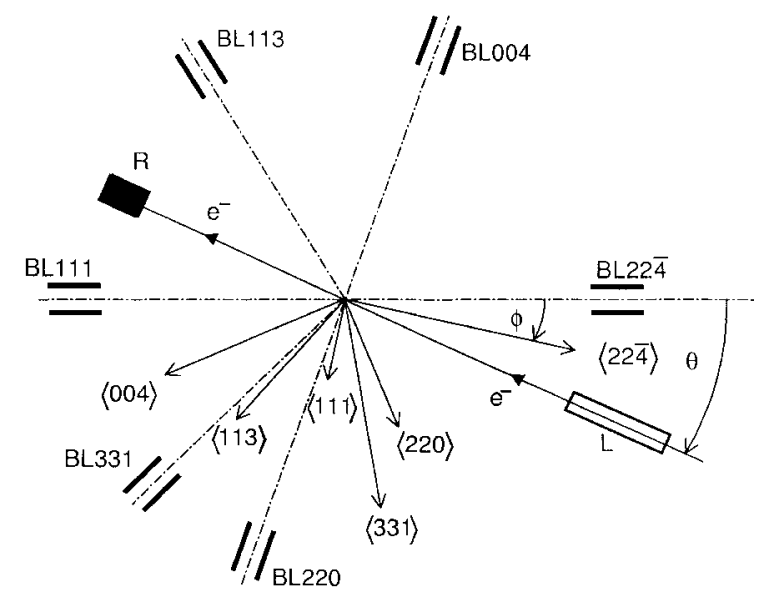

Fig. 3. The multi-X-ray beam facility based on a diamond-like crystal radiator with the $\langle 2 \overline{2} 0\rangle$ axis perpendicular to the facility plane. The crystal radiator is not shown and its alignment is indicated by reciprocal lattice vectors. Several reciprocal lattice vectors $\vec{g}=\langle 111\rangle,\langle 220\rangle,\langle 113\rangle,\langle 004\rangle,\langle 331\rangle,\langle 22 \overline{4}\rangle$ lie in the facility plane. Corresponding X-ray beamlines BL111, BL220, BL113, BL004, BL331, BL224 are in the vicinity of Bragg directions for these vectors at angles of $180^{\circ}, 109.5^{\circ}, 239^{\circ}, 289.5^{\circ}$, $136^{\circ}$ and $0^{\circ}$, respectively. The linac $\mathrm{L}$ is on the rotating platform. Smooth tuning of X-ray energies in the beamlines is possible with linac and crystal-radiator rotations, provided $\theta=2 \phi$. For example, the X-ray energies in the above-mentioned Bragg directions are 9.5, 4.4, 12.5, 6.7, 8.9 and $5.7 \mathrm{keV}$, respectively, for the $\mathrm{Si}$ crystal radiator and $\theta=2 \phi=24^{\circ}$. The electron beam, being passed through the crystal radiator, can be used for irradiation of consumer R situated on the other side of the same rotating platform.
As an example, Fig. 3 shows the scheme of a multi-X-ray beam facility for a diamond-like crystal with the axis $\langle 2 \overline{2} 0\rangle$ perpendicular to the facility plane. The beamlines BL are installed in the vicinity of corresponding immobile Bragg directions. The beamline can pass the whole PXR reflection or one of its maxima to a consumer. Usually, the PXR yield maxima with parallel polarizations are at angles $\pm \gamma_{\text {eff }}^{-1}$ relative to the Bragg direction in the facility plane, and the maxima with perpendicular polarizations lie at the same angles in the plane perpendicular to the facility plane. Ge, $\mathrm{Si}$ or diamond crystals can be used in the facility shown in Fig. 3. The PXR spectral peak energies usually are practically independent of the electron beam energy at $E_{\mathrm{e}}>10 \mathrm{MeV}$. Tuning of PXR spectral peak energies is performed simultaneously in all beamlines BL at rotations provided $\theta=2 \phi$. The facility can be used as a synchronous multichannel X-ray sweep generator.

\section{Discussion}

The above-described estimations demonstrate good prospects for the PXR-based X-ray beam in calibration of space X-ray telescopes at energies from a few $\mathrm{keV}$ up to hundreds of $\mathrm{keV}$, including $511 \mathrm{keV}$. The application of PXR can quicken the procedure of telescope calibration.

Note some problems. The estimations were performed by the kinematic theory. This theory had been confirmed experimentally for PXR energies up to tens of $\mathrm{keV}$ at electron beam energies from several $\mathrm{MeV}$ to about a thousand $\mathrm{MeV}$. In our estimations, we partially considered the case of PXR energies up to hundreds of $\mathrm{keV}$, generated at a moderate electron beam energy of about 100 $\mathrm{MeV}$ to provide the appropriate X-ray beam diameter. However, the yield of such a hard PXR in this particular case has not been sufficiently studied by experiments. Besides, several factors that may be of importance in this case were not taken into account in the present first estimations. In particular, the role of multiple electron scattering and of possible interference between the PXR and the coherent/ordinary bremsstrahlung is not known clearly in this case. The most efficient way 
to solve these problems seems to perform detailed experimental investigations of hard PXR at energies of hundreds of $\mathrm{keV}$ generated by an electron beam of moderate energy (about $100 \mathrm{MeV}$ ) from the appropriate linac at laboratory conditions.

The setup can provide $\gamma$-ray beam with energy exceeding $1 \mathrm{MeV}$. At $\theta=0$ (see Fig. 1) the linac axis coincides to the beamline axis. Therefore coherent bremsstrahlung from a crystalline target can be used as a source of $\gamma$-ray beam. The channeling radiation can be used in this geometry too. Properties of such photon beams strongly depend on electron beam energy.

Above, we have described the estimations of hard X-ray beam properties at $E_{\gamma}>10 \mathrm{keV}$ and $\theta \leqslant 0.3 \mathrm{rad}$ for the Laue geometry only. Note briefly the setup capabilities for generation of softer X-rays. The X-ray beam at energies of several $\mathrm{keV}$ can be provided in the same way using the same setup at greater values of $\theta$. The Bragg geometry may appear more convenient for the purpose. At angles $\theta>25^{\circ}$, the use of PXR with a perpendicular polarization may be more preferential, as it provides a higher yield (see calculations in Figs. 9.19 and 9.20 in [8]). The PXR from crystalline radiators at energies of several $\mathrm{keV}$ has been studied experimentally. Besides, the sub-keV PXR energies can be generated from a layered radiator with an appropriate period, instead of a crystalline one. A layered X-ray mirror can be used as a radiator. However, as far as we know, experiments on observation of sub-keV PXR still have not been performed.

The development of the PXR-based monochromatic polarized tunable $\mathrm{X}$-ray beam generator can give impetus to a wider application of X-ray beams, because a linac of moderate energy, even situated on a rotating platform, is much cheaper than a synchrotron source. Of particular use here can be the generation of hard X-rays. The range of $\mathrm{X}$-ray beam energies covers all atomic energies of hard elements (see Fig. 2).

A multi-X-ray beam facility, similar to the one shown in Fig. 3, can be used for the development of X-ray technologies in the regions, where there are no operating synchrotron sources, for example in Ukraine. It can provide users with several relatively inexpensive tunable X-ray beams for various applications and investigations.

In future, the PXR-based generators of X-ray beams, similar to ones described here, can be used for the development of X-ray technologies in outer space. Launching and operation of a linac in the space seems to be provided much easier as compared to a storage ring.

\section{Acknowledgements}

This paper became possible partially due to Project \#1031 from the Science and Technology Center in Ukraine.

\section{References}

[1] Ya.B. Fainberg, N.A. Khizhnyak, Zh. Ehksp. Teor. Fiz. 32 (1957) 883.

[2] M.L. Ter-Mikaelian, High-Energy Electromagnetic Processes in Condensed Media, Wiley, New York, 1972.

[3] S.A. Vorobiev, B.N. Kalinin, S. Pak, A.P. Potylitsin, Pis'ma Zh. Ehksp. Teor. Fiz. 41 (1985) 3.

[4] A.V. Shchagin, V.I. Pristupa, N.A. Khizhnyak, Phys. Lett. A 148 (1990) 485 .

[5] R.B. Fiorito, D.W. Rule, X.K. Maruyama, K.L. DiNova, S.J. Evertson, M.J. Osborne, D. Snyder, H. Rietdyk, M.A. Piestrup, A.H. Ho, Phys. Rev. Lett. 71 (1993) 704.

[6] S. Asano, I. Endo, M. Harada, S. Ishii, T. Kobayashi, T. Nagata, M. Muto, K. Yoshida, H. Nitta, Phys. Rev. Lett. 70 (1993) 3247.

[7] X. Artru, P. Rullhusen, Nucl. Instr. and Meth. B 145 (1998) 1.

[8] A.V. Shchagin, X.K. Maruyama, Parametric X-rays, in: S.M. Shafroth, J.C. Austin (Eds.), Accelerator-Based Atomic Physics Technique and Applications, AIP Press, New York, 1997, p. 279. For correction about PXR polarization, see [10].

[9] A.V. Shchagin, Phys. Lett. A 262 (1999) 383.

[10] A.V. Shchagin, Phys. Lett. A 247 (1998) 27.

[11] R.B. Fiorito, D.W. Rule, M.A. Piestrup, Q. Li, A.H. Ho, X.K. Maruyama, Nucl. Instr. and Meth. B 79 (1993) 758.

[12] A.V. Shchagin, V.I. Pristupa, N.A. Khizhnyak, Nucl. Instr. and Meth. B 99 (1995) 227.

[13] A.V. Shchagin, N.A. Khizhnyak, Nucl. Instr. and Meth. B 119 (1996) 115. 